\title{
Polarised Drell-Yan process in COMPASS
}

\author{
Márcia Quaresma*† \\ On behalf of the COMPASS Collaboration \\ LIP - Laboratório de Instrumentação e Física Experimental de Partículas, Av. Elias Garcia \\ 14-1, 1000-149 Lisboa, Portugal \\ E-mail: marcia@lip.pt
}

\begin{abstract}
The COMPASS collaboration at CERN has been contributing to the knowledge of the nucleon structure and its description in terms of the transverse momentum dependent PDFs, accessed through the SIDIS process. In 2014/2015 the data taking was dedicated to the polarised Drell-Yan (DY) measurement with a negative pion beam and a transversely polarised proton target, as well as unpolarised nuclear targets. These data are now being analysed. One of the main goals is to verify the predicted sign change of the Sivers distribution function when accessed from DY with respect to SIDIS. The details on the experimental setup, the covered phase space, a preliminary first look into data distributions and predictions for the full data sample will be presented.
\end{abstract}

XXIV International Workshop on Deep-Inelastic Scattering and Related Subjects

11-15 April, 2016

DESY Hamburg, Germany

\footnotetext{
*Speaker.

$\dagger$ This work was supported by the Portuguese Fundação para a Ciência e a Tecnologia (CERN/FIS-NUC/0017/2015).
} 


\section{Introduction}

The COMPASS experiment at CERN has been contributing to the knowledge of the nucleon structure, which is described by eight Transverse Momentum Dependent (TMD) Parton Distribution Functions (PDFs) at leading twist, if the intrinsic transverse momentum, $k_{T}$, of the quarks inside the nucleon is taken into account. The TMD PDFs can be accessed in COMPASS either from Semi Inclusive Deep Inelastic Scattering (SIDIS) or from Drell-Yan (DY) measurements. The theoretical prediction of the Sivers and Boer-Mulders TMD PDFs sign change when extracted from these two processes [1] will be checked using the COMPASS data. Its confirmation is considered a crucial test of the QCD TMD approach. The DY process is an excellent tool to access TMD PDFs at COMPASS, since the QCD TMD approach is valid in the covered phase space, where dimuon mass is much larger than the dimuon transverse momentum. In addition all of the TMD PDFs are expected to be sizeable in the valence quark region, which is dominant in COMPASS.

\section{DY and SIDIS processes}

The DY and SIDIS cross-sections are expressed in terms of angular modulations and the amplitude of each modulation contains a convolution of two PDFs in the DY case, one corresponding to the quark from the pion beam and the other to the quark from the nucleon target. In the SIDIS case each amplitude contains a convolution of one PDF and one Fragmentation Function (FF). The experimental extraction of these amplitudes is done through a measurement of azimuthal asymmetries between events coming from the target cells with opposite polarisations. The DY cross section in terms of these asymmetries, keeping only contributions from leading twist TMD PDFs is

$$
\begin{aligned}
& \frac{d \sigma^{D Y}}{d^{4} q d \Omega}=\frac{\alpha^{2}}{\Phi q^{2}} \hat{\sigma}_{U}\left\{\left(1+\cos ^{2}(\theta)+\sin ^{2}(\theta) A_{U U}^{\cos (2 \phi)} \cos (2 \phi)\right)\right. \\
& +S_{T}\left[(1+\cos (\theta)) A_{U T}^{\sin \left(\phi_{S}\right)} \sin \left(\phi_{S}\right)\right. \\
& \left.\left.+\sin ^{2}(\theta)\left(A_{U T}^{\sin \left(2 \phi+\phi_{S}\right)} \sin \left(2 \phi+\phi_{S}\right)+A_{U T}^{\sin \left(2 \phi-\phi_{S}\right)} \sin \left(2 \phi-\phi_{S}\right)\right)\right]\right\}
\end{aligned}
$$

where $\Phi=4 \sqrt{\left(P_{a} \cdot P_{b}\right)^{2}-M_{a}^{2} M_{b}^{2}}$ and $\hat{\sigma}_{U}=F_{U U}^{1}$. Here $\phi_{S}$ is the azimuthal angle of the nucleon polarisation in the target rest frame with $z$ axis along the beam momentum and the $x$ axis along the direction of the transverse momentum of the produced dimuon; $\phi$ and $\theta$ are azimuthal and polar angles in the Collins-Soper frame, a virtual photon rest frame, where the $z$ axis is the bisector of the angle between the beam momentum and the target momentum, and $x$ axis is defined in the plane of beam and target momenta. The asymmetries, $A$, are defined as amplitudes of the azimuthal modulation defined in the superscript; and the two subscripts correspond to the polarisation of the beam and of the target, "U" stands for unpolarised and "T" transversely polarised. The SIDIS cross section in terms of the azimuthal asymmetries and keeping only contributions from leading twist TMD PDFs and FFs reads 


$$
\begin{aligned}
& \frac{d \sigma^{S I D I S}}{d x d y d z d \psi d \phi_{h} d P_{h T}^{2}}=\frac{\alpha^{2}}{x y Q^{2}} \frac{y^{2}}{2(1-\varepsilon)}\left(1+\frac{\gamma^{2}}{2 x}\right) \sigma_{U}\left\{1+\varepsilon \cos \left(2 \phi_{h}\right) A_{U U}^{\cos \left(2 \phi_{h}\right)}\right. \\
& +S_{T}\left[\sin \left(\phi_{h}-\phi_{S}\right) A_{U T}^{\sin \left(\phi_{h}-\phi_{S}\right)}+\varepsilon \sin \left(\phi_{h}+\phi_{S}\right) A_{U T}^{\sin \left(\phi_{h}+\phi_{S}\right)}\right. \\
& \left.\left.+\varepsilon \sin \left(3 \phi_{h}-\phi_{S}\right) A_{U T}^{\sin \left(3 \phi_{h}-\phi_{S}\right)}\right]\right\}
\end{aligned}
$$

where $\varepsilon=\left(1-y-1 / 4 \gamma^{2} y^{2}\right) /\left(1-y+1 / 2 y^{2}+1 / 4 \gamma^{2} y^{2}\right), \gamma=2 M x / Q$ and $\sigma_{U}=F_{U U, T}$. Here $\phi_{h}$ is the azimuthal angle of the produced hadron and $\phi_{S}$ is the azimuthal angle of the target spin vector; these angles are defined in the target rest frame with $z$ axis along the virtual photon momentum and $x$ axis along the lepton transverse momentum, where transverse is meant with respect to the $z$ axis.

\begin{tabular}{|c|c|c|}
\hline DY & Proton TMD PDF & SIDIS \\
\hline$A_{U U}^{\cos (2 \phi)} \propto h_{1, \pi}^{\perp q} \otimes h_{1, p}^{\perp q}$ & Boer-Mulders & $A_{U U}^{\cos \left(2 \phi_{h}\right)} \propto h_{1}^{\perp q} \otimes H_{1 q}^{\perp h}$ \\
\hline$A_{U T}^{\sin \left(\phi_{S}\right)} \propto f_{1, \pi}^{q} \otimes f_{1 T, p}^{\perp q}$ & Sivers & $A_{U T}^{\sin \left(\phi_{h}-\phi_{S}\right)} \propto f_{1 T}^{\perp q} \otimes D_{1 q}^{h}$ \\
\hline$A_{U T}^{\sin \left(2 \phi-\phi_{S}\right)} \propto h_{1, \pi}^{\perp q} \otimes h_{1, p}^{q}$ & transversity & $A_{U T}^{\sin \left(\phi_{h}+\phi_{S}\right)} \propto h_{1}^{q} \otimes H_{1 q}^{\perp h}$ \\
\hline$A_{U T}^{\sin \left(2 \phi+\phi_{S}\right)} \propto h_{1, \pi}^{\perp q} \otimes h_{1 T, p}^{\perp q}$ & pretzelosity & $A_{U T}^{\sin \left(3 \phi_{h}-\phi_{S}\right)} \propto h_{1 T}^{\perp q} \otimes H_{1 q}^{\perp h}$ \\
\hline
\end{tabular}

Table 1: Leading Order asymmetries in DY and SIDIS and the TMD PDFs.

There is a direct correspondence between the asymmetries originating from the same nucleon TMD PDFs in the two cases as shown in Table 1. COMPASS has already published results on the Sivers asymmetry using the SIDIS data collected in 2007 and 2010 [2]. These data pointed to a difference between COMPASS and HERMES results, HERMES measurement giving a slightly higher Sivers asymmetry. This difference was attributed to the different $Q^{2}$ phase space coverage of the two experiments. For a better comparison between DY and SIDIS results from COMPASS, the SIDIS analysis was performed in different $Q^{2}$ ranges. One of them, $Q^{2}>16(\mathrm{GeV} / c)^{2}$, coincides with the DY high mass range $\left(M_{\mu \mu}>4 \mathrm{GeV} / c^{2}\right)$, where the asymmetries extraction is going to be performed. The DY and SIDIS phase-space overlap at large $Q^{2}$ as shown in Fig. 1.

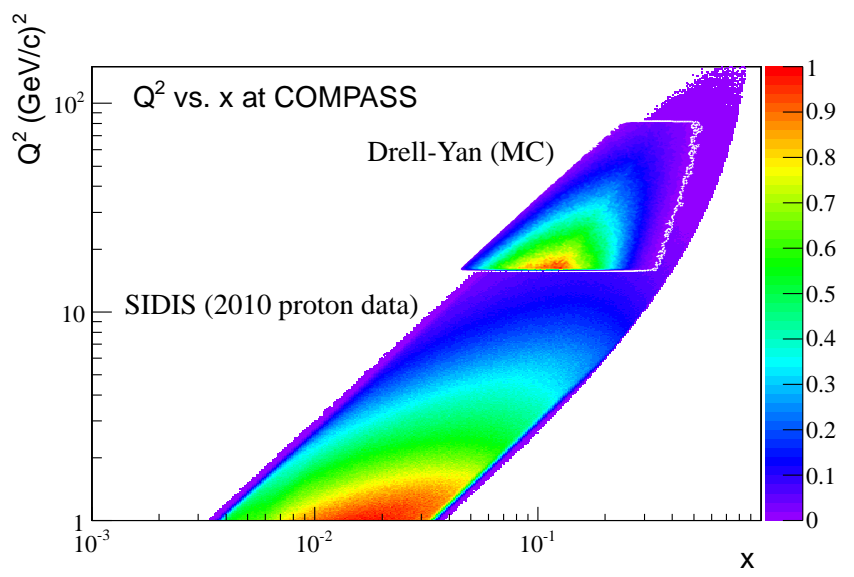

Figure 1: $Q^{2}$ versus $x$ distribution for DY high mass events and for SIDIS events. 


\section{Sivers asymmetry}

The SIDIS data were analysed in four different $Q^{2}$ ranges. For each range events were binned in $x, z, p_{T}$ and $W$. Results for Sivers asymmetry are shown in Fig. 2 for positive and negative hadrons, four kinematic variables and four $Q^{2}$ intervals, selecting only events with $z>0.2$. For largest $Q^{2}$ the asymmetry is positive and increases with $x$ for positive hadrons, while for negative hadrons it is compatible with zero, except for one $x$ bin.

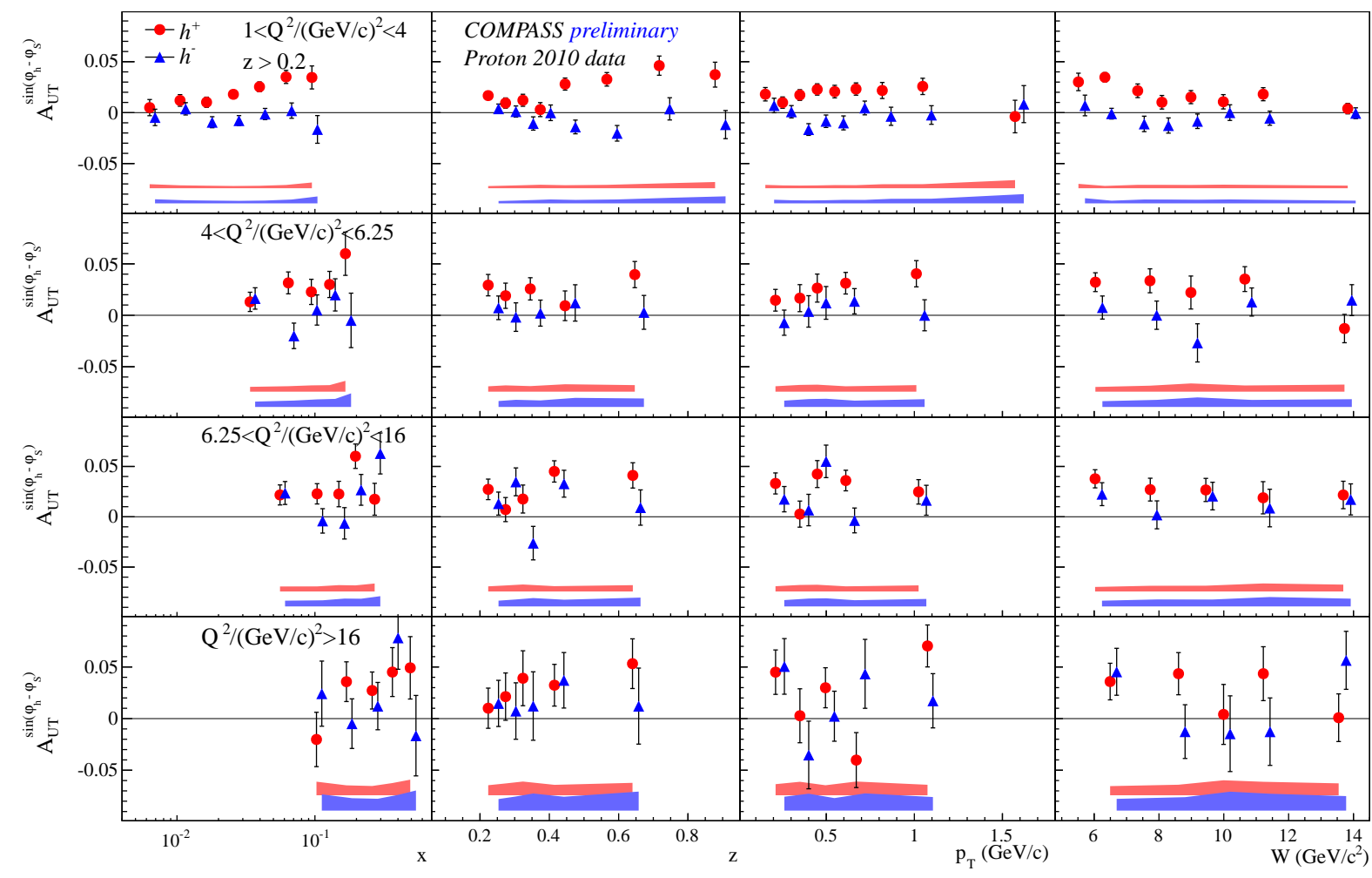

Figure 2: Sivers asymmetry for four $Q^{2}$ intervals in bins of $x, z, p_{T}$ and $W$.

\section{Polarised DY programme}

Polarised DY data taking took place in 2015, being the first ever polarised DY measurement in a fixed target experiment. A negative pion beam with momentum $190 \mathrm{GeV} / c$ impinging on two ammonia target cells with opposite transverse polarisation was used. For the DY study several changes were performed in the setup of the COMPASS spectrometer. The most important was the installation of a hadron absorber downstream the target with the purpose to stop the secondary hadrons produced along with the DY process as well as the non interacting beam. The DY muons when passing through the absorber undergo multiple scattering, which degrades the resolution of the interaction point position, $Z_{v t x}$. The latter is of a major importance since the two cells are polarised in opposite directions and it is mandatory to know from which cell the event is coming. 
In order to improve this resolution a vertex detector was installed in the first part of the absorber, as shown in Fig. 3. In addition to the polarised ammonia target, a $7 \mathrm{~cm}$ aluminium target was introduced in the absorber central open space along the beam line before the beginning of the tungsten beam plug, which is also used as a target. The unpolarised data from these targets will be analysed and COMPASS aims to contribute with studies on the flavour dependent EMC effect, the modification of quarks distributions in nuclei, and also dedicated studies on the Lam-Tung sum rule.

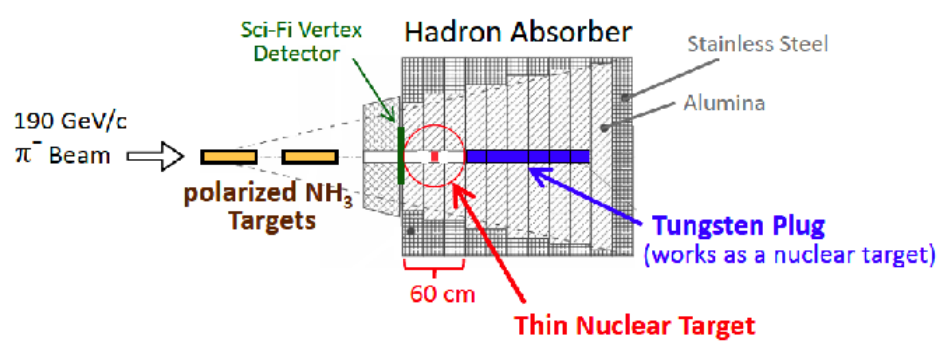

Figure 3: Hadron absorber sketch.

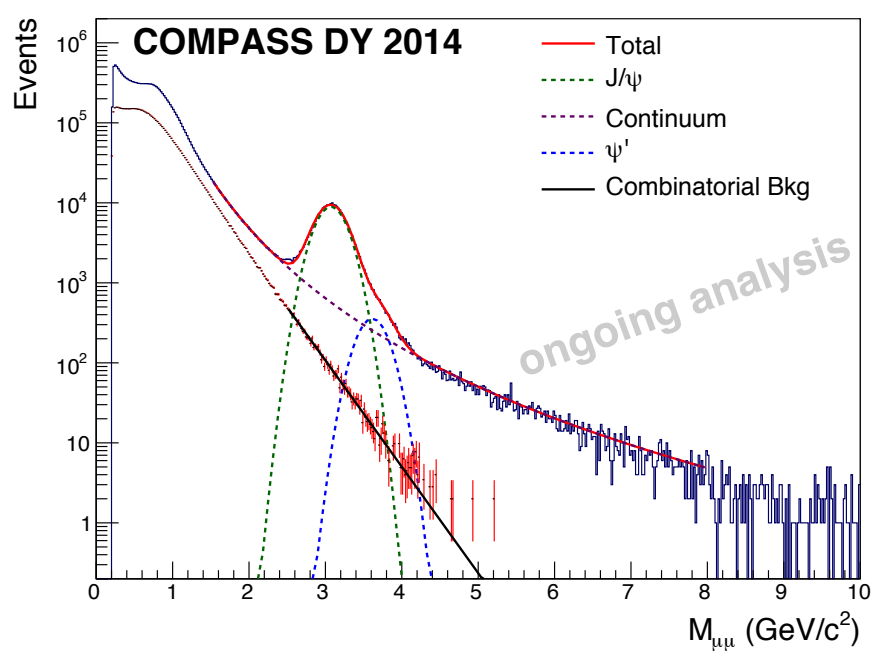

Figure 4: Dimuon mass distribution.

In the end of 2014 a DY pilot run took place. The ammonia cells were not yet polarised and the beam intensity was $30 \%$ lower than in 2015 . However this pilot run was important for the commissioning of the spectrometer. The ongoing analysis of the data is based on seventeen days of stable data taking. The dimuon mass distribution is shown in Fig. 4. The two broken curves mark the two resonances, $\mathrm{J} / \psi$ and $\psi^{\prime}$ (green and blue online), respectively. The combinatorial background measurements are also marked (red points online), which extends to approximately 5 $\mathrm{GeV} / c^{2}$. The high mass DY events, $M_{\mu \mu}>4 \mathrm{GeV} / c^{2}$, are almost background free and will be used for the extraction of the asymmetries. The $\mathrm{Z}_{v t x}$ distribution for these events is shown in Fig. 5 . 
A clear signal from all targets is visible, pointing to a reasonable resolution in $\mathrm{Z}_{v t x}$, even though the vertex detector was not working properly during the data taking and the reconstruction of these data did not benefit from it.

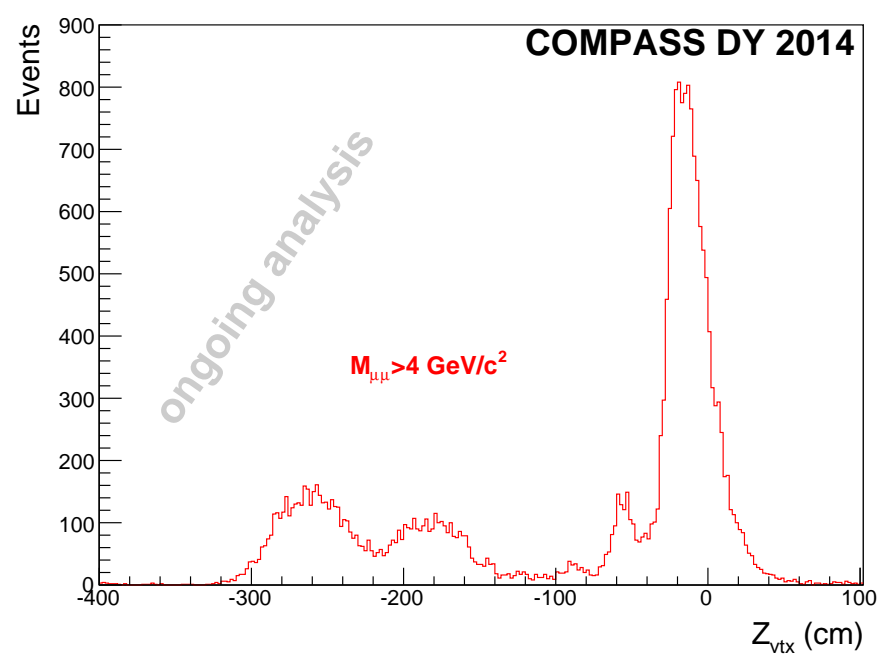

Figure 5: $Z_{v t x}$ distribution for muon pairs with $M_{\mu \mu}>4 \mathrm{GeV} / c^{2}$.

The distribution of $x_{2}$ versus $x_{1}$, the momentum fraction carried by the quark within the proton and the quark within the pion, respectively, is shown in Fig. 6. Obviously the COMPASS experiment provides access to the valence quarks distributions.

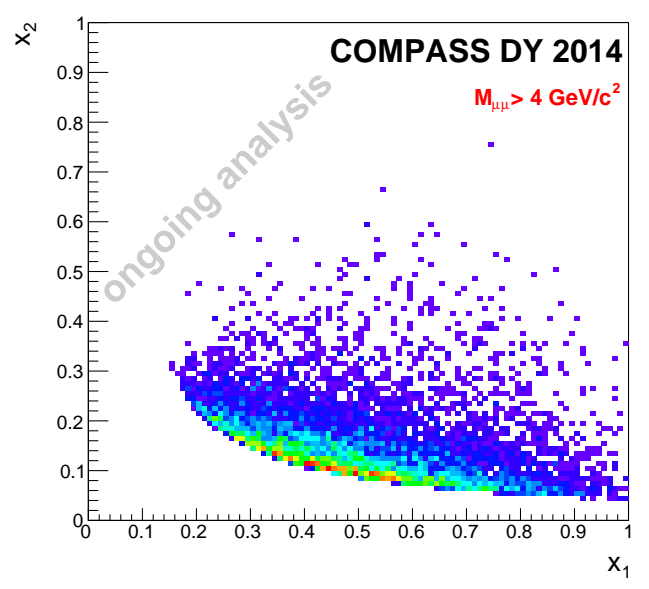

Figure 6: Distribution of $x_{2}$ versus $x_{1}$ for muon pairs with $M_{\mu \mu}>4 \mathrm{GeV} / c^{2}$.

In 2015 data from four months of stable running with polarized ammonia target were taken. Preliminary analysis of these data indicate that a statistical uncertainty of $\sim 2.8 \%$ for the Sivers asymmetry is achieved, while some existing models predict an asymmetry up to $10 \%$, as shown in Figs. 7 and 8. 


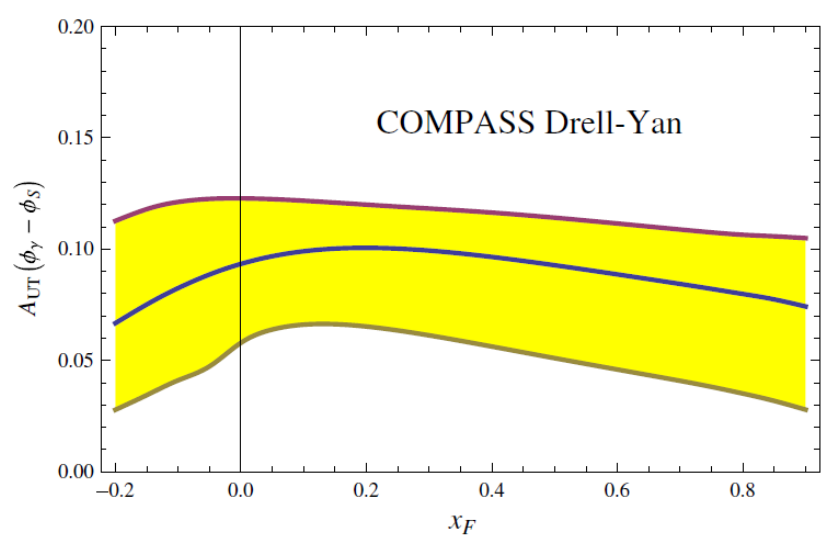

Figure 7: Prediction for the Sivers asymmetry for COMPASS DY data. Here $x_{F}=x_{1}-x_{2}$ and a cut $p_{T}<2$ $\mathrm{GeV} / c$ was applied [3].

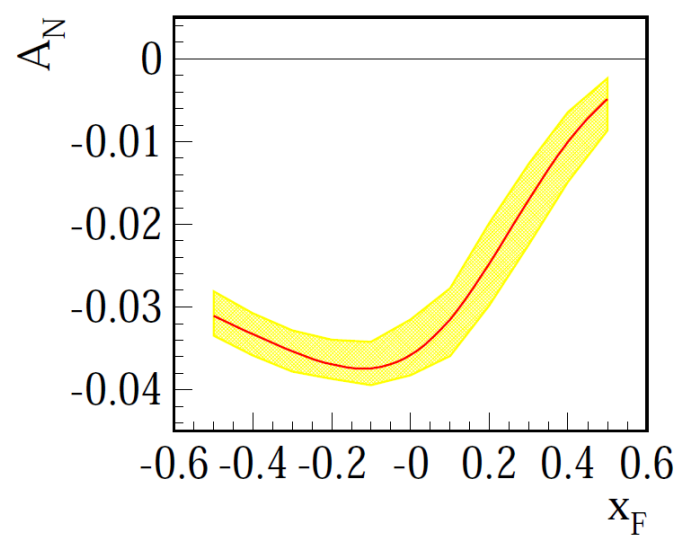

Figure 8: Prediction for the Sivers asymmetry for COMPASS DY data. Here $x_{F}=x_{2}-x_{1}$ and a cut $p_{T}<1$ $\mathrm{GeV} / c$ was applied [4].

These data together with the SIDIS data offer a unique opportunity to extract the TMD PDFs from both processes in the same experiment and to have a fundamental test of the predicted Sivers sign change.

\section{References}

[1] J.C. Collins, Phys. Lett. B536 (2002) 43.

[2] COMPASS Collaboration, C. Adolph et al., Phys. Lett. B744 (2015) 250.

[3] P. Sun and F. Yuan, Phys. Rev. D88 (2013) 114012.

[4] M.G. Echevarria et al., Phys. Rev. D89 (2014) 074013. 